\title{
Editorial
}

\section{The miracle of brain neuropeptides: targeting the brain oxytocin system is a true treatment option in numerous psychiatric disorders including schizophrenia}

Dozens of neuropeptides of the brain act in concert to regulate all aspects of behaviour and physiological functions, including modulatory effects on classical neurotransmitter systems. For example, neuropeptides are involved in the fine-tuned regulation of emotional and social behaviours, with some of them exerting anxiolytic effects such as cholecystokinin-8 (CCK-8), prolactin, neuropeptide $\mathrm{Y}$, the recently discovered neuropeptide $S$, as well as oxytocin, and others having opposite effects, thus increasing anxiety levels such as corticotropin releasing factor (CRF) or vasopressin. From an evolutionary point of view, both a certain level of anxiety and fear responsiveness (to avoid danger), as well as a certain degree of risk taking behaviour (to discover food resources and mating partners), need to be in perfect balance underlining the important regulatory capacity of these neuropeptides. In addition to emotional behaviours, various neuropeptides are key modulators of social behaviours closely linked to emotionality and, therefore, summarised as socio-emotional behaviours.

In this context, there are lots of scientific and clinical interests in the neuropeptide oxytocin, which acts both as a blood neurohormone after its secretion from the neurohypophysis, but also as a brain neurotransmitter/neuromodulator when released within distinct brain regions. This central release can occur in several ways, for example, from dendrites and perikarya within the hypothalamus, or from axon terminals in limbic target regions. The presence of oxytocin receptors in hypothalamic and limbic regions, of which only one has been identified to date, is the essential basis for its multiple socioemotional behaviours. In addition to its anxiolytic actions, which have been shown in the amygdala and the hypothalamic paraventricular nucleus in rodents $(1,2)$, oxytocin promotes prosocial behaviours - many of them being in a reproductive context such as maternal care and defence, mother-offspring bonding, sexual behaviour or pair-bonding (for review see 3-5). In addition, oxytocin plays a role in many non-reproduction-related social behaviours such as social recognition (6), social preference (7) and fear extinction (8).

These findings together with the potential of OXT to attenuate depression-related symptoms (9) triggered a plethora of human studies with the aim to pharmacologically target the brain oxytocin system thus modulating emotional and physiological stress coping, and social behaviours. Hereby, the method of choice to increase oxytocinergic neurotransmission in the human brain is via intranasal application under the assumption that oxytocin is taken up into the brain compartment (10). Multiple effects of intranasally applied oxytocin on (11) various behavioural parameters and on neuronal activity patterns as shown by functional magnet resonance imaging (fMRI) studies confirm the high potential of this psychopharmacotherapy also for clinical use (for review see 12,13). Just to provide a few examples, intranasal oxytocin makes humans more trusting (14), even under conditions of betrayal (15), improves the learning performance especially when reinforced by social cues, and increases emotional empathy in men (to the level found in women which was not affected by nasal oxytocin) (16). To further underline the prosocial effects of oxytocin, it was shown that oxytocin-treated subjects make more eye movements towards the eye region when viewing human faces (17), and show an improved ability to read the mental state of others using social cues from facial expressions (18). Further, after oxytocin treatment anxiety levels in response to psychosocial 
stress (19), and neuronal responses to fearful faces in the amygdala (20) were suppressed.

These studies mainly performed in healthy male volunteers have been recently complemented by findings of positive effects of oxytocin in patients with autism spectrum disorder $(21,22)$. In this context it should be mentioned that a link between autism spectrum disorders and polymorphisms in the oxytocin receptor gene and changes in oxytocin availability have been described (for review see 12,13).

The review by MacDonald and Feifel in the present issue of Archives of Neuropsychiatria Scandinavia summarises existing evidence for the potential of oxytocin as psychopharmacotherapy in schizophrenia. Schizophrenia is characterised by severe socio-emotional dysfunctions which, in most cases, prevent the continuation of an independent life. The currently available treatment options include psychosocial intervention and antipsychotic pharmacotherapies which mainly target the dopamine (D2 receptor antagonism) and 5-HT (5-HT2A antagonism) systems. Therefore, it is exciting that two independent double-blind, placebo-controlled US studies could show promising first results with intranasal oxytocin in schizophrenic patients $(23,24)$.

In their review, MacDonald and Feifel provide a comprehensive overview of the existing preclinical and clinical findings on the potential use of oxytocin as an antipsychotic drug. Thus, they outline the preclinical experimental evidence for antipsychotic-like effects of oxytocin, which should mainly involve interactions with the dopaminergic (reward) system of the nucleus accumbens and striatum, but also the glutamatergic system. It is of note that in various experimental settings oxytocin was able to restore social deficits, e.g. after prenatal stress-induced reduction of oxytocin expression (25), or after social defeat-induced lack of social preference (7) (for review see 5). Experimentally, the best model of schizophrenia is the experimental induction of deficits in prepuls inhibition (PPI), and in rats oxytocin was able to restore PPI functions (26).

On the basis of these data, the brain oxytocin system is likely to be altered in patients suffering from schizophrenia, with altered plasma or cerebrospinal fluid (CSF) oxytocin levels or morphometric data indicating perturbations in the (central) oxytocin system. However, as is the case also for other psychopathologies, where brain oxytocin seems to participate in its etiology such as depression (9) or anxiety-related diseases including social phobia (13), the difficulty exists to monitor the brain oxytocin system of these patients, or at least to correlate plasma parameters with the central neuropeptide system (27).

After the first clinical trials, which were performed in the former Soviet Union in the 1960s and 1970s with oxytocin applied intravenously or intramuscularly (25 IU), there was a break of 30 years before clinical assessment of the effects of oxytocin were restarted with a series of well-designed clinical trials. In 2010, Feifel et al. (23) applied oxytocin twice daily over 3 weeks to a cohort of schizophrenic patients adjunctive to standard antipsychotic therapy. In the third week of treatment a significant, although, minor improvement of several symptoms appeared, among them verbal memory. A year later, Pedersen et al. (24) published a modified protocol, i.e. a shorter treatment duration (2 weeks). Again, oxytocin produced some therapeutic effects and improved the Positive and Negative Syndrome Scale (PANSS score), which was mainly due to a more pronounced improvement in various social deficit symptoms such as restoration of the ability of social judgement and cognition in oxytocin- versus placebo-treated patients. These promising studies are supported by single-dose studies in schizophrenic patients showing that oxytocin improved emotion and social cognitive functions. In all these studies, intranasal oxytocin seems well tolerated and safe.

All those fascinated by the multiple behavioural functions of the oxytocin and vasopressin neuropeptide families and their underlying genetic and neurobiological mechanisms follow the development of the human use of oxytocin with great interest, as this system proved to be a true example for translational neuroscience. However, if we aim to develop intranasal oxytocin treatment as an established therapeutic option (alone or possibly in combination with other pharmacotherapies) for patients suffering from schizophrenia, social phobia, depression or autism, we need to invest substantial efforts into the neurobiology of nasally applied neuropeptides. In this context, we need to reveal (a) routes and mechanisms of uptake of oxytocin into the brain compartment, (b) parenchymal diffusion or local accumulation, (c) possible inhibitory or stimulatory interactions with the endogenous oxytocin system including oxytocin receptor up- or down-regulation, as well as (d) gender-dependent and/or genetically determined variance in oxytocin responsiveness due to differences in oxytocin receptor distribution and binding.

In conclusion, oxytocin receptor-mediated actions are importantly involved in processing socioemotional stimuli with a certain level of activity of the endogenous oxytocin system essential for protecting against emotional or social dysfunctions. The review by MacDonald and Feifel further emphasises that the brain oxytocin system seems to play an antipsychotic role possibly by protecting against the disruption of mesolimbic dopaminergic circuitries. Thus, increasing the availability of bioactive (though synthetic) oxytocin within the extracellular fluid of 
the brain by nasal application and, thus, increasing the general activity of the brain oxytocin system may compensate for possible dysfunctions in the endogenous oxytocin system as suggested for various psychopathologies such as social phobia, depression, autism as well as schizophrenia.

Inga D. Neumann

Department of Behavioural and Molecular Neurobiology, University of Regensburg, Regensburg, Germany

\section{References}

1. Bale Tl, Davis AM, Auger AP, Dorsa DM, McCarthy MM. CNS region-specific oxytocin receptor expression: importance in regulation of anxiety and sex behavior. J Neurosci 2001;21:2546-2552.

2. Blume A, Bosch OJ, Miklos $\mathrm{S}$ et al. Oxytocin reduces anxiety via ERK1/2 activation: local effect within the rat hypothalamic paraventricular nucleus. Eur J Neurosci 2008;27:1947-1956.

3. Bosch OJ, Neumann ID. Both oxytocin and vasopressin are mediators of maternal care and aggression in rodents: from central release to sites of action. Horm Behav 2011;61:293-303.

4. Donaldson ZR, Young LJ. Oxytocin, vasopressin, and the neurogenetics of sociality. Science 2008;322:900-904.

5. Neumann ID. The advantage of social living: brain neuropeptides mediate the beneficial consequences of sex and motherhood. Front Neuroendocrinol 2009;30:483-496.

6. Pfaff DW, Rapin I, Goldman S. Male predominance in autism: neuroendocrine influences on arousal and social anxiety. Autism Res 2011;4:163-176.

7. Lukas M, Toth I, Reber SO, Slattery DA, Veenema AH, Neumann ID. The neuropeptide oxytocin facilitates pro-social behavior and prevents social avoidance in rats and mice. Neuropsychopharmacology 2011;36:2159-2168.

8. Toth I, Neumann ID, Slattery DA. Social fear conditioning: a novel and specific animal model to study social anxiety disorder. Neuropsychopharmacology 2012;37:1433-1443.

9. Slattery DA, Neumann ID. Oxytocin and major depressive disorder: experimental and clinical evidence for links to aetiology and possible treatment. Pharmaceuticals 2010;3:702-724.

10. Born J, Lange T, Kern W, McGregor GP, Bickel U, FEHM HL. Sniffing neuropeptides: a transnasal approach to the human brain. Nat Neurosci 2002;5:514-516.

11. Chang SW, Barter JW, Ebitz RB, Watson KK, Platt ML. Inhaled oxytocin amplifies both vicarious reinforcement and self reinforcement in rhesus macaques (Macaca mulatta). Proc Natl Acad Sci U S A 2012;109:959-964.

12. Bartz JA, ZaKi J, Bolger N, Ochsner KN. Social effects of oxytocin in humans: context and person matter. Trends Cogn Sci 2011;15:301-309.
13. Meyer-Lindenberg A, Domes G, Kirsch P, Heinrichs M. Oxytocin and vasopressin in the human brain: social neuropeptides for translational medicine. Nat Rev Neurosci 2011;12:524-538.

14. Kosfeld M, Heinrichs M, ZaK PJ, Fischbacher U, FeHR E. Oxytocin increases trust in humans. Nature 2005; 435:673-676.

15. Baumgartner T, Heinrichs M, Vonlanthen A, FisCHBACHER U, FEHR E. Oxytocin shapes the neural circuitry of trust and trust adaptation in humans. Neuron 2008; 58:639-650.

16. Hurlemann R, Patin A, Onur OA et al. Oxytocin enhances amygdala-dependent, socially reinforced learning and emotional empathy in humans. J Neurosci 2010; 30:4999-5007.

17. Gamer M, Zurowski B, Buchel C. Different amygdala subregions mediate valence-related and attentional effects of oxytocin in humans. Proc Natl Acad Sci U S A 2010; 107:9400-9405.

18. Domes G, Heinrichs M, Michel A, Berger C, Herpertz SC. Oxytocin improves "mind-reading" in humans. Biol Psychiatry 2007;61:731-733.

19. Heinrichs M, Baumgartner T, Kirschbaum C, EHLERT U. Social support and oxytocin interact to suppress cortisol and subjective responses to psychosocial stress. Biol Psychiatry 2003;54:1389-1398.

20. Kirsch P, Esslinger C, Chen Q et al. Oxytocin modulates neural circuitry for social cognition and fear in humans. J Neurosci 2005;25:11489-11493.

21. Andari E, Duhamel JR, Zalla T, Herbrecht E, Leboyer M, Sirigu A. Promoting social behavior with oxytocin in high-functioning autism spectrum disorders. Proc Natl Acad Sci U S A 2010;107:4389-4394.

22. Guastella AJ, Einfeld SL, Gray KM et al. Intranasal oxytocin improves emotion recognition for youth with autism spectrum disorders. Biol Psychiatry 2010;67: 692-694.

23. Feifel D, Macdonald K, Nguyen A et al. Adjunctive intranasal oxytocin reduces symptoms in schizophrenia patients. Biol Psychiatry 2010;68:678-680.

24. Pedersen CA, Gibson CM, Rau SW et al. Intranasal oxytocin reduces psychotic symptoms and improves Theory of mind and social perception in schizophrenia. Schizophr Res 2011;132:50-53.

25. Lee PR, Brady DL, Shapiro RA, Dorsa DM, Koenig JI. Prenatal stress generates deficits in rat social behavior: Reversal by oxytocin. Brain Res 2007;1156:152-167.

26. Feifel D, Reza T. Oxytocin modulates psychotomimeticinduced deficits in sensorimotor gating. Psychopharmacology (Berl) 1999;141:93-98.

27. Landgraf R, Neumann ID. Vasopressin and oxytocin release within the brain: a dynamic concept of multiple and variable modes of neuropeptide communication. Front Neuroendocrinol 2004;25:150-176. 